\title{
Ab Initio Prediction of Threshold Displacement Energies in $\mathrm{ZrC}$
}

\author{
Ming-Jie Zheng ${ }^{\mathrm{a}, \mathrm{b}, \mathrm{c}}$, Izabela Szlufarska ${ }^{\mathrm{a}, \mathrm{d}}$, Dane Morgan ${ }^{\mathrm{a}, \mathrm{d}, *}$ \\ ${ }^{a}$ Department of Materials Science and Engineering, University of Wisconsin- Madison, \\ Madison, Wisconsin, U.S.A. \\ ${ }^{b}$ Institute of Nuclear Energy Safety Technology, Chinese Academy of Sciences, China \\ ${ }^{c}$ Key Laboratory of Neutronics and Radiation Safety, Chinese Academy of Sciences, \\ China \\ ${ }^{d}$ Materials Science Program, University of Wisconsin-Madison, Madison, Wisconsin, \\ U.S.A. \\ * Corresponding author's email:ddmorgan@wisc.edu
}

\begin{abstract}
The threshold displacement energies $\left(E_{d}\right)$ of $\mathrm{C}$ and $\mathrm{Zr}$ atoms in $\mathrm{ZrC}$ have been determined using $a b$ initio molecular dynamics simulation. The values of $E_{d}$ have been predicted along the three main high-symmetry crystallographic directions [001], [011], and [111], and the averaged $E_{d}$ values are $16 \mathrm{eV}$ and $37 \mathrm{eV}$ for the $\mathrm{C}$ and $\mathrm{Si}$ sublattices, respectively.
\end{abstract}


We further explore the dependence of $E_{d}$ on small deviations from these high-symmetry directions and on the presence of $\mathrm{C}$ vacancies, which are often encountered in $\mathrm{ZrC}_{x}$ alloys. The trends in values are explained in terms of the structural and chemical properties of $\mathrm{ZrC}$. The predicted $E_{d}$ values provide relevant parameters for future modeling of radiation damage in $\mathrm{ZrC}$.

\section{Introduction}

Zirconium carbide $(\mathrm{ZrC})$, due to its high temperature stability, oxygen gettering ability, chemical inertness and resistance to irradiation damage[1-3], is of interest as a radiation resistant structural material in the high temperature gas cooled reactor (HTGCR). However, the radiation responses of $\mathrm{ZrC}$ are still poorly understood, including both its high-temperature microstructural changes [4] and the origin of its superior resistance to amorphization. In fact, radiation-induced amorphization of $\mathrm{ZrC}$ has not yet been observed experimentally at any temperature or dose [1, 4-10]. One of the key unknown parameters needed to model the irradiation response of $\mathrm{ZrC}$, e.g., using SRIM simulations[11], is the threshold displacement energy $\left(E_{d}\right)$.

$E_{d}$ has several different definitions [12-15] and in this work we follow Ref. [15] and it is defined as the minimum kinetic energy required to produce a permanent defect on the same sublattice as the PKA, such as a stable Frenkel pair, which may or may not involve displacement of the PKA. Please see the Appendix for results following a 
somewhat different definition. The Kinchin-Pease and Norgett-Robinson-Torrens (NRT) models for Frenkel pair defect production under irradiation predict that Frenkel pairs will be produced by a high-energy ion in a quantity inversely proportional to the value of $E_{d}$ [16]. The widely used SRIM code [11] for predicting defect production rates also requires $E_{d}$ values as input. In addition, in some cases the $E_{d}$ values have been associated with a material's amorphization resistance[17]. For example, in a molecular dynamics (MD) simulation of ion beam irradiation on GaN[18], the high $E_{d}$ values were used to help explain the small initial damage and high dose to amorphization of $\mathrm{GaN}$. It is possible that $E_{d}$ values in $\mathrm{ZrC}$ (if they are sufficiently high) could be responsible for the unusual resistance of this material to amorphization. It is also of fundamental interest to explore if the unusual structural aspects of $\mathrm{ZrC}$, in which $\mathrm{C}$ behaves almost like an interstitial and which can tolerate a very large number of $\mathrm{C}$ vacancies (a large $x$ in $\mathrm{ZrC}_{x}$ ), produces unusual values of $E_{d}$ compared to nominally similar materials, e.g., SiC. In general $E_{d}$ is not isotropic, which means that it can have different values for different crystallographic directions and for symmetry distinct atoms.

The value of $E_{d}$ is difficult to measure experimentally, since a single defect is hard to create in irradiation experiments, and even if it can be generated, it is difficult to identify [12]. Among the computational methods, the molecular dynamics (MD) technique has been proved to be an effective and efficient way to predict threshold displacement energies [12, 19-20]. MD simulations can be performed using classical 
interatomic potentials (which we will call Classical MD (CMD)) or ab initio calculations (which we will call $A b$ Initio MD (AIMD)). Although AIMD is far slower and more computationally demanding than CMD, it avoids the use of interatomic potentials, which have been suggested to be the largest source of errors in $E_{d}$ values determined with CMD [21]. AIMD has previously been successfully applied to study $E_{d}$ in $\mathrm{SiC}$ [12], GaN [20], and $\mathrm{Y}_{2} \mathrm{Ti}_{2} \mathrm{O}_{7}[22]$.

Threshold displacement energies in $\mathrm{ZrC}$ have been previously estimated by Gosset et al. [5] who found $E_{d}$ to be equal to $25 \mathrm{eV}$ and $35 \mathrm{eV}$ for $\mathrm{C}$ and $\mathrm{Zr}$, respectively. However, these values were estimated based on experimentally observed values in $\mathrm{TaC}$ rather than in $\mathrm{ZrC}$. $E_{d}$ for $\mathrm{ZrC}$ has not been measured or calculated directly. Furthermore, no information about the anisotropy of $E_{d}$ or the possible influence of $\operatorname{ZrC}_{x}(x<1)$ offstoichiometry on $E_{d}$ is known.

In this work, we use AIMD simulation to calculate the $E_{d}$ in $\mathrm{ZrC}$. To explore anisotropy, we predict $E_{d}$ along different directions for the primary knock-on atom (PKA), considering displacements both directly along high-symmetry and slightly off highsymmetry directions. We also investigate effects of the off-stoichiometric properties of $\mathrm{ZrC}_{x}(0.6<x<0.98)[2]$ by predicting the influence of $\mathrm{C}$ vacancies on $E_{d}$.

\section{Methods}


The AIMD simulations were performed with the Vienna Ab-initio Simulation Package (VASP)[23-26] in the framework of density functional theory (DFT) and the projector-augmented wave (PAW) method. The exchange-correlation was treated in the generalized gradient approximation (GGA), as parameterized by Perdew, Burke, and Ernzerhof (PBE)[27]. The PAW potentials were generated with the following valence electronic configurations: $5 s 4 d 5 p$ for $\mathrm{Zr}$ and $2 s^{2} 2 p^{2}$ for C. An energy cut-off of $450 \mathrm{eV}$ was used.

We used a $3 \times 3 \times 3$ cubic supercell of $\mathrm{ZrC}$ containing 216 atoms. The system-size convergence was verified by comparing the corresponding $E_{d}$ values in a 64-atom cell with those in a 216-atom cell, as shown in Table A.1 in the Appendix. It was found that the values agree within $5 \mathrm{eV}$ except for $\mathrm{Zr}[001]$, where the 64 atom cell gives a large error due to a periodic boundary effect. This agreement suggests that the results for the 216-atom cell are well converged with respect to size to within a few eV or less. The large discrepancy for $E_{d}(\mathrm{Zr}[001])$ in these two supercells is due to a "wrapping" error in the 64-atom cell, where the collision wraps across the periodic cell and the energy from the PKA effectively interacts with itself, causing a large reduction of $E_{d}$. No such obvious "wrapping" effects occurred for the 216-atom supercell simulations. In all AIMD runs, we use a single gamma point for the k-points.

Three steps are used for the AIMD simulations. In the first step, the system is equilibrated at $300 \mathrm{~K}$ using a Nose-Hoover thermostat (the equilibration runs). The time 
step is $1 \mathrm{fs}$ and the equilibration time lasts $1 \mathrm{ps}$. In the second step, a series of initial kinetic energies are added to the PKA to attempt to generate Frenkel pairs (the production runs). No thermostat is used in this step. The time step is $0.1 \mathrm{fs}$, which was found to give a maximum displacement during one AIMD time step of about $0.02 \AA$, as measured in simulations for C PKA with an initial kinetic energy of $50 \mathrm{eV}$. We believe this time step is small enough as this associated maximum displacement is lower than the maximum displacement of $0.1 \AA$ recommended by Corrales et al.[28] for calculations of excess energy in displacement cascades. After simulating for about $0.2 \mathrm{ps}$, we are able to observe whether a Frenkel pair will form or not at the given kinetic energy and thereby roughly estimate the value of $E_{d}$. To determine $E_{d}$, a velocity corresponding to the kinetic energy $\left(E_{k}\right)$ was given to a $\mathrm{C}$ (or Zr) PKA atom. The PKA velocity was initially aligned along the three main crystallographic directions: [001], [011], and [111], as shown in Figs. 1 (a) and (b). We have also checked the effects of tilting the velocity direction slightly off the high-symmetry directions (by a small angle of about $\alpha \approx 2^{\circ}$ ). To define the tilted directions, we use the unit vector triplet $\left(\mathbf{e}_{\mathrm{x}}, \mathbf{e}_{\mathrm{y}}, \mathbf{e}_{\mathrm{z}}\right)$ to describe the velocity directions. As we know, the unit vectors along the [001], [011], and [111] are $(0,0,1),\left(0, \sin 45^{\circ}\right.$, $\left.\cos 45^{\circ}\right)$, and $\left(\sin 55^{\circ} \cos 45^{\circ}, \sin 55^{\circ} \sin 45^{\circ}, \cos 55^{\circ}\right)$, respectively. The corresponding unit vectors along the [001]_tilted, [011]_tilted, and [111]_tilted $\operatorname{are}\left(0, \sin 2^{\circ}, \cos 2^{\circ}\right),(0$, $\left.\sin 47^{\circ}, \cos 43^{\circ}\right)$, and $\left(\sin 57^{\circ} \cos 45^{\circ}, \sin 57 \sin 45^{\circ}, \cos 53^{\circ}\right)$, respectively. 
(a)

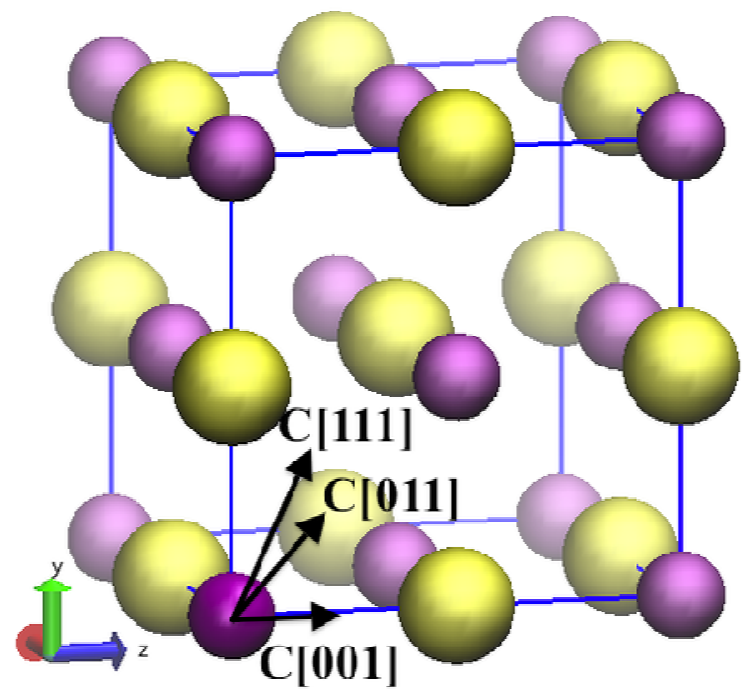

(c)

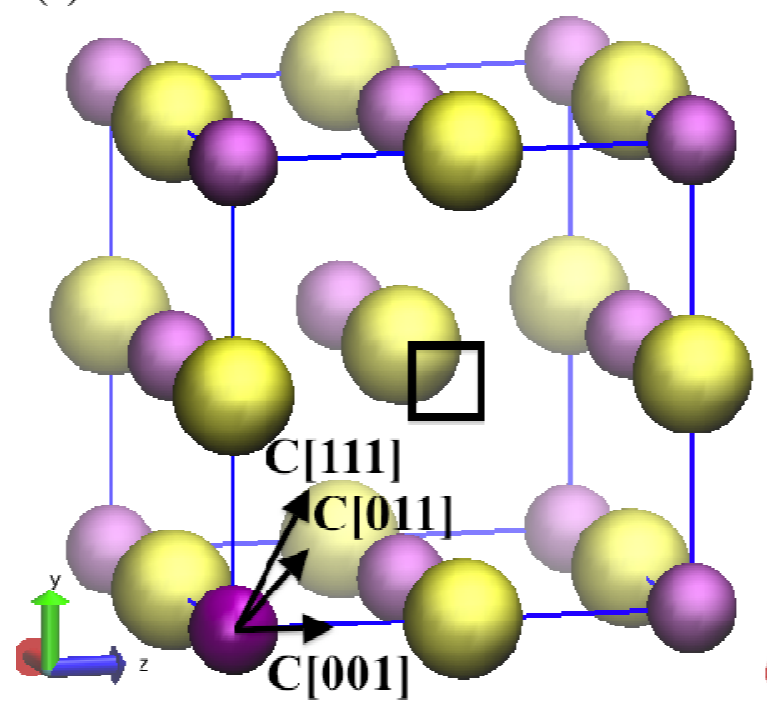

(b)

$\operatorname{Zr}[111]$

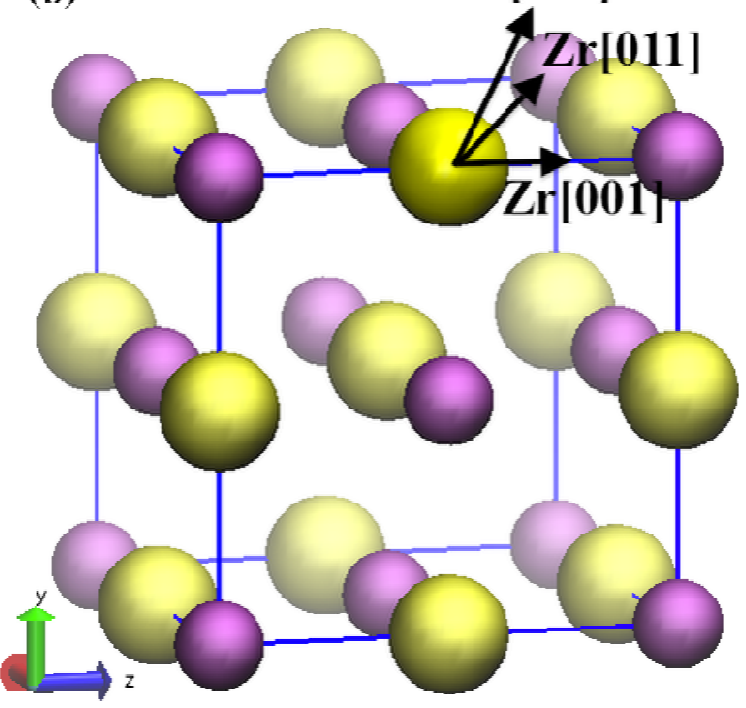

(d)

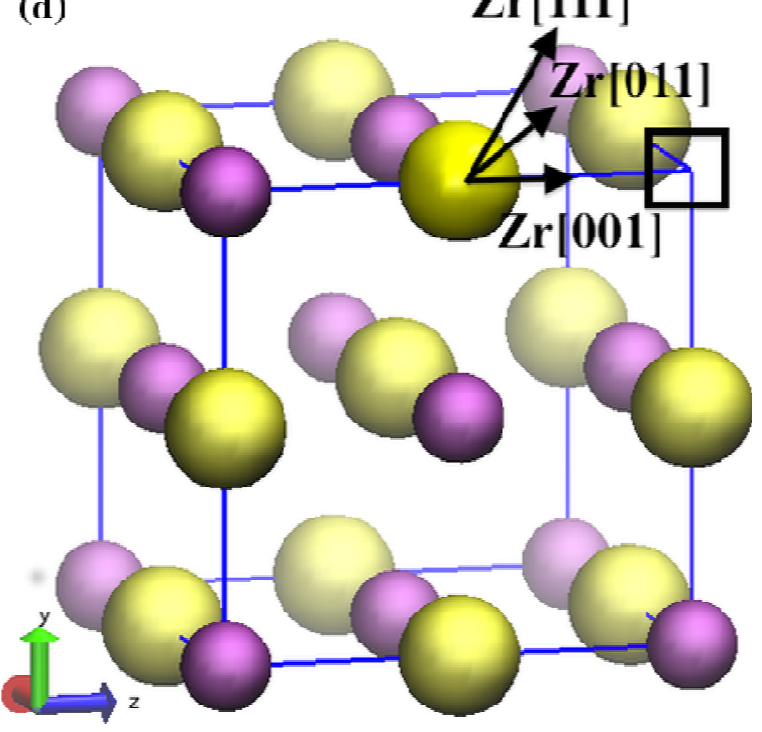

Figure 1. Configurations of (a) $\mathrm{C}$ as PKA in $\mathrm{ZrC}$ crystal, (b) $\mathrm{Zr}$ as PKA in $\mathrm{ZrC}$ crystal, (c) $\mathrm{C}$ as $\mathrm{PKA}$ in $\mathrm{ZrC}$ with one $\mathrm{C}$ vacancy, and (d) $\mathrm{Zr}$ as $\mathrm{PKA}$ in $\mathrm{ZrC}$ with one $\mathrm{C}$ vacancy. The high-symmetry crystallographic directions of displacement are labeled with the atom and direction of displacement. For example, $\mathrm{C}[001]$ means to displace $\mathrm{C}$ atom along the [001] direction. 
In the third step in the AIMD simulations, for the initial kinetic energies around the estimate $E_{d}$ from step two, longer time simulations with a time step of $1 \mathrm{fs}$ with a thermostat at $300 \mathrm{~K}$ are performed for the evolution of produced defects (the evolution runs). The stability of Frenkel pairs is tested for about 3 ps. If a stable Frenkel pair is formed in AIMD simulations, the system is then subsequently relaxed at $0 \mathrm{~K}$ to reach the stable configuration to ensure that the Frenkel pair is stable. To determine $E_{d}$, simulations are performed for each kinetic energy $\left(E_{k}\right)$ value given to the PKA atom and $E_{d}$ is set at the lowest value where a Frenkel pair is formed.

Initially all three steps are performed for each case on a course grid of energies with an energy interval of $5 \mathrm{eV}$ in order to approximately determine $E_{d}$, which requires at most 10 simulations (one at each $E_{k}$ value). Then about five runs with an energy interval of 1 $\mathrm{eV}$ around the approximate $E_{d}$ determined from the coarse grid are conducted to improve the precision so that the value where the Frenkel pair is formed is accurate to within $1 \mathrm{eV}$. Once the value for $E_{d}$ is known, a final longer time AIMD simulation (for about $1 \mathrm{ps}$ ) is checked to make sure the Frenkel pair is stable. This $1 \mathrm{eV}$ range of possible values is then used as the estimated errors bars in Tables 1 and 2.

\section{Results and Discussion}




\section{1. $\quad E_{d}$ for impulses along the high-symmetry crystallographic directions}

Table 1. Threshold displacement energies in $\mathrm{ZrC}$ and $\mathrm{SiC}$ (SiC from Ref.[14]), along the high-symmetry crystallographic directions (the [ $[\overline{1} \overline{1} \overline{1}]$ direction is equivalent to the [111] direction in $\mathrm{ZrC}$ ). The heading "Defect" refers to the final Frenkel pair defect structure formed. $\mathrm{V}_{\mathrm{C}}$ and $\mathrm{V}_{\mathrm{Zr}}$ are a $\mathrm{C}$ vacancy and $\mathrm{Zr}$ vacancy, respectively. $\mathrm{Zr}_{\mathrm{Td}}$ is a $\mathrm{Zr}$ interstitial located in a $\mathrm{Zr}$-centered double tetrahedron. $d_{\mathrm{FP}}$ is the distance between the interstitial (the middle $\mathrm{C}$ atom of the $\mathrm{C}-\mathrm{C}-\mathrm{C}$ trimer, or closer $\mathrm{Zr}$ atom of the $\mathrm{Zr}$ - $\mathrm{Zr}$ dumbbell) and the vacancy of the Frenkel pair. The error bar of $1 \mathrm{eV}$ is determined as described in Section 2.

\begin{tabular}{|c|c|c|c|c|}
\hline Direction & $\begin{array}{c}E_{d} \text { in } \mathrm{ZrC}(\mathrm{eV}) \\
216 \text { atoms }\end{array}$ & $\begin{array}{c}E_{d} \text { in } \mathrm{SiC}(\mathrm{eV}) \\
1000 \text { atoms }[14]\end{array}$ & Defect & $d_{\mathrm{FP}}\left(\mathbf{a}_{0}\right)$ \\
\hline $\mathrm{C}[001]$ & $19 \pm 1$ & 20.0 & $\mathrm{~V}_{\mathrm{C}}+\mathrm{C}-\mathrm{C}-\mathrm{C}$ trimer & 0.47 \\
\hline $\mathrm{C}[011]$ & $20 \pm 1$ & 22.5 & $\mathrm{~V}_{\mathrm{C}}+\mathrm{C}-\mathrm{C}-\mathrm{C}$ trimer & 0.47 \\
\hline $\mathrm{C}[111]$ & $7 \pm 1$ & 20.5 & $\mathrm{~V}_{\mathrm{C}}+\mathrm{C}-\mathrm{C}-\mathrm{C}$ trimer & 0.47 \\
\hline $\mathrm{C}[\overline{1} \overline{1} \overline{1}]$ & $7 \pm 1$ & 47.5 & $\mathrm{~V}_{\mathrm{C}}+\mathrm{C}-\mathrm{C}-\mathrm{C}$ trimer & 0.47 \\
\hline $\begin{array}{l}\text { C sublattice } \\
\text { weighted } \\
\text { average }\end{array}$ & $16 \pm 1$ & 25.5 & & \\
\hline $\operatorname{Zr}[001]$ & $71 \pm 1$ & 49.5 & $\begin{array}{c}\mathrm{V}_{\mathrm{Zr}}+\mathrm{Zr}-\mathrm{Zr} \text { dumbbell \& } \\
2 \mathrm{~V}_{\mathrm{C}}+\mathrm{C}-\mathrm{C}-\mathrm{C} \text { trimer }+ \\
\mathrm{C}-\mathrm{C} \text { dumbbell }\end{array}$ & 0.82 \\
\hline $\operatorname{Zr}[011]$ & $22 \pm 1$ & 70.0 & $\mathrm{~V}_{\mathrm{Zr}}+\mathrm{Zr}-\mathrm{Zr}$ dumbbell & 1.20 \\
\hline $\operatorname{Zr}[111]$ & $33 \pm 1$ & 105.0 & $\begin{array}{c}\mathrm{V}_{\mathrm{Zr}}+\mathrm{Zr}_{\mathrm{Td}} \& \\
\mathrm{~V}_{\mathrm{C}}+\mathrm{C}-\mathrm{C}-\mathrm{C} \text { trimer }\end{array}$ & 0.81 \\
\hline
\end{tabular}




\begin{tabular}{ccccc}
$\mathrm{Zr}[\overline{\mathrm{1}} \overline{1} \overline{1}]$ & $33 \pm 1$ & 62.0 & $\mathrm{~V}_{\mathrm{Zr}}+\mathrm{Zr}_{\mathrm{Td}} \&$ & \\
$\begin{array}{c}\text { Zr sublattice } \\
\text { weighted } \\
\text { average }\end{array}$ & $37 \pm 1$ & 69.4 & \\
\hline \hline
\end{tabular}

Table 1 and Fig. 2 report the results of $E_{d}$ of $\mathrm{C}$ and $\mathrm{Zr}$ atoms along the main highsymmetry crystallographic directions in $\mathrm{ZrC}$. Results for $\mathrm{SiC}[12,14]$ are also included for comparison. We include directions [111] and [ $[\overline{1} \overline{1} \overline{1}]$ as they are different in SiC, although they are equivalent for $\mathrm{ZrC}$. We note that as the structures of $\mathrm{ZrC}$ and $\mathrm{SiC}$ are rocksalt and zincblend, respectively, the different directions do not correspond to the same environments. Therefore, differences in $E_{d}$ between the two systems may be due to both their different chemistries and their different structures, and any interpretation of the origin of these differences must at least take both possibilities into account. It is shown that $E_{d}$ is strongly anisotropic. It is easiest to displace $\mathrm{C}$ atom along the [111] direction $\left(E_{d}(\mathrm{C}[111]) \approx 7 \pm 1 \mathrm{eV}\right)$ and most difficult along the [011] direction $\left(E_{d}(\mathrm{C}[011]) \approx 20 \pm 1\right.$ $\mathrm{eV}$ ). This ordering can be rationalized in terms of the structure and chemistry of $\mathrm{ZrC}$. In particular, the low value along the [111] direction can be explained by the fact that when the C PKA was displaced along this direction it moves into a region of significant open space and rapidly begins to form bonds with the second nearest-neighbor $\mathrm{C}$ atoms before colliding with the third nearest-neighbor $\mathrm{Zr}$ atom. This bonding effect, which stabilizes the [111] direction, does not occur in the other directions. Along the [011] direction, the 
C PKA kicks out its second nearest neighbor $\mathrm{C}$ atom along the [011] direction, the $\mathrm{C}$ PKA goes back to its original lattice site, and the kicked-out $\mathrm{C}$ atom forms a $\mathrm{C}-\mathrm{C}$ dumbbell with the sixth nearest neighbor $\mathrm{C}$ atom of the original PKA site. Along the [001] direction $\left(E_{d}(\mathrm{C}[001]) \approx 19 \pm 1 \mathrm{eV}\right)$, the $\mathrm{C}$ PKA impacts a $\mathrm{Zr}$ atom, which rebounds the $\mathrm{C}$ atom backward due to the much greater mass of $\mathrm{Zr}$. The returning $\mathrm{C}$ atom passes near its original lattice site, and then it deviates further from the lattice site while it vibrates back and forth. Eventually, this $\mathrm{C}$ atom becomes a $\mathrm{C}$ interstitial and diffuses to its neighboring atoms and forms a stable $\mathrm{C}$ Frenkel pair.

The average $E_{d}$ values reported in Table 1 correspond to the weighted average calculated over the four high-symmetry directions. The weighting factors take into account the number of physically distinct but symmetry equivalent directions in $\mathrm{ZrC}$,

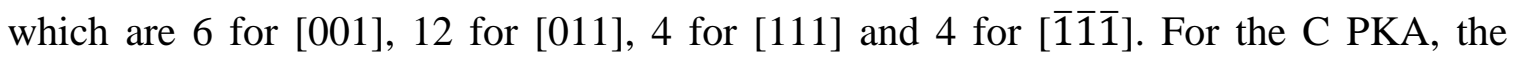
weighted average value of $E_{d}$ is $16 \pm 1 \mathrm{eV}$. For all cases of C displacement, C Frenkel pairs rather than $\mathrm{Zr}$ Frenkel pairs are formed at the end of the MD simulation, presumably in part due to the fact that the C PKA is displaced most dramatically, and perhaps in part due to the much lower formation energies of $\mathrm{C}$ Frenkel pairs than $\mathrm{Zr}$ ones [3, 7]. The $\mathrm{C}$ Frenkel pairs at the end of MD runs are fully relaxed to obtain their stable configuration. The $\mathrm{C}$ interstitial (the C-C-C trimer) is not far from the $\mathrm{C}$ vacancy site. The interstitialvacancy separation $d_{\mathrm{FP}}$, which is defined as the distance from the $\mathrm{C}$ interstitial atom at the middle of the C-C-C trimer to the vacancy lattice site (i.e., the position that the $\mathrm{C}$ 
occupied before it was removed), is about $0.47 a_{0}$, where $a_{0}=4.72 \AA$ is the lattice constant of $\mathrm{ZrC}$ unit cell.

For the $\mathrm{Zr}$ atom, the easiest direction to displace the atom is along the [011] direction $\left(E_{d}(\operatorname{Zr}[011]) \approx 22 \pm 1 \mathrm{eV}\right)$, while the hardest direction is along the [001] direction $\left(E_{d}(\operatorname{Zr}[001]) \approx 71 \pm 1 \mathrm{eV}\right)$. In the [011] direction, the Zr PKA kicks out its second nearest-neighbor $\mathrm{Zr}$ atom and replaces it. After fully relaxation, a stable $\mathrm{Zr}$ FP with the interstitial-vacancy separation $d_{\mathrm{FP}} \approx 1.20 a_{0}$ is formed (the distance is measured from the closer $\mathrm{Zr}$ of the $\mathrm{Zr}-\mathrm{Zr}$ dumbbell to the $\mathrm{Zr}$ vacancy site). Along the [001] direction, the Zr PKA kicks out its first nearest-neighbor $\mathrm{C}$ atom, which forms a $\mathrm{C}-\mathrm{C}$ dumbbell. Then the $\mathrm{Zr}$ PKA forms a $\mathrm{Zr}$-Zr dumbbell and kicks out another $\mathrm{C}$ atom which forms a C-C-C trimer. Thus one $\mathrm{Zr}$ FP with $d_{\mathrm{FP}} \approx 0.82 a_{0}$ and two additional C FPs are formed. In the [111] direction $\left(E_{d}(\operatorname{Zr}[111]) \approx 33 \pm 1 \mathrm{eV}\right)$ the displaced $\mathrm{Zr}$ PKA kicks out its third nearest-neighbor $\mathrm{C}$ atom, which forms a C-C-C trimer . The $\mathrm{Zr}$ PKA moves to the interstitial site of $\mathrm{Zr}$-centered double tetrahedron. After fully relaxation, a stable $\mathrm{Zr}$ FP with the interstitial-vacancy separation $d_{\mathrm{FP}} \approx 0.81 a_{0}$ is formed.

To explore the possible correlation between the amorphization resistance and $E_{d}$, we compare our calculated $E_{d}$ in $\mathrm{ZrC}$ with $E_{d}$ in $\mathrm{SiC}$, determined using similar AIMD methods in Ref. [12]. These two systems might show important differences as $\mathrm{ZrC}$ has much higher amorphization resistance than $\mathrm{SiC}$ [8]. As shown in Table 1 and Fig. 2, the averaged $E_{d}$ values on $\mathrm{Si}$ and $\mathrm{C}$ sublattices are about $25.5 \mathrm{eV}$ and $69.4 \mathrm{eV}$, respectively, 
compared to $16 \pm 1 \mathrm{eV}$ and $37 \pm 1 \mathrm{eV}$ on the $\mathrm{Zr}$ and $\mathrm{C}$ sublattices. Thus the values of $E_{d}$ are actually lower in $\mathrm{ZrC}$ than those in $\mathrm{SiC}$ (especially along the directions $\mathrm{C}[\overline{1} \overline{1} \overline{1}]$, $\mathrm{Zr} / \mathrm{Si}[011]$ and $\mathrm{Zr} / \mathrm{Si}[111])$, despite the greater resistance to amorphizatin of $\mathrm{ZrC}$. These results indicate that the values of $E_{d}$ have little to do with the fact that $\mathrm{SiC}$ can be readily amorphized [29-32] while $\mathrm{ZrC}$ cannot [8].

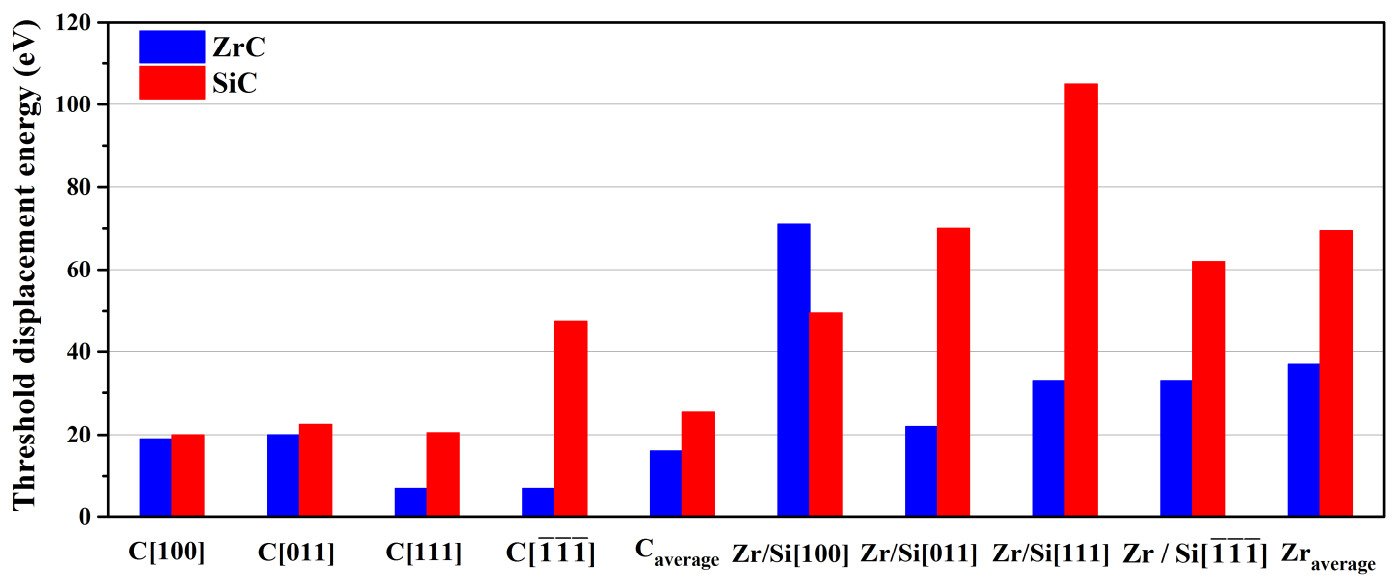

Figure 2. The comparison of $E_{d}$ values on the $\mathrm{C}$ and $\mathrm{Zr}(\mathrm{Si}$ ) sublattices in $\mathrm{ZrC}$ (blue) and that in $\mathrm{SiC}$ (red), along each of the high-symmetry crystallographic directions as well as the weighted average sublattice values. SiC values are from Ref.[14].

\section{2. $E_{d}$ for impulses just off the high-symmetry crystallographic directions}

Since $E_{d}$ values are highly anisotropic, we now check the dependence of $E_{d}$ on tilting by a small angle off the high-symmetry crystallographic directions. Comparing $E_{d}$ along the exact crystallographic directions with those along the corresponding tilted directions, the energy changes are within the uncertainty range of $1 \mathrm{eV}$, as shown in Table 
2. The overall small differences indicate that there is no obvious effect on $E_{d}$ by slightly tilting the PKA velocity direction off the high-symmetry crystallographic directions. This is expected from the fact that the PKA atom vibrates around its lattice site at room temperature, which already breaks any symmetry of the PKA atom displacement. Therefore, there is no significant difference between the impulse direction along the highsymmetry crystallographic direction or slightly off this direction.

Table 2. Threshold displacement energies for $\mathrm{ZrC}$ along the exact or tilted highsymmetry crystallographic directions and with a $\mathrm{C}$ vacancy $\left(\mathrm{V}_{\mathrm{C}}\right)$. The error bar of $1 \mathrm{eV}$ is determined as described in Section 2.

\begin{tabular}{lccc}
\hline Direction & $\boldsymbol{E}_{\boldsymbol{d}}$ perfect $(\mathbf{e V})$ & $\boldsymbol{E}_{d_{-} \text {itted }}$ perfect $(\mathbf{e V})$ & $\boldsymbol{E}_{\boldsymbol{d}}$ with $\mathbf{V}_{\mathbf{C}}(\mathbf{e V})$ \\
\hline $\mathrm{C}[001]$ & $19 \pm 1$ & $19 \pm 1$ & $20 \pm 1$ \\
$\mathrm{C}[011]$ & $20 \pm 1$ & $21 \pm 1$ & $21 \pm 1$ \\
$\mathrm{C}[111]$ & $7 \pm 1$ & $7 \pm 1$ & $11 \pm 1$ \\
$\mathrm{C}$ sublattice & & & \\
weighted average & $16 \pm 1$ & $16 \pm 1$ & $18 \pm 1$ \\
\hline Zr[001] & $71 \pm 1$ & $72 \pm 1$ & $71 \pm 1$ \\
Zr[011] & $22 \pm 1$ & $21 \pm 1$ & $21 \pm 1$ \\
Zr[111] & $33 \pm 1$ & $34 \pm 1$ & $34 \pm 1$ \\
Zr sublattice & & & $37 \pm 1$ \\
weighted average & $37 \pm 1$ & $37 \pm 1$ & \\
\hline \hline
\end{tabular}




\section{3. $E_{d}$ in the presence of $\mathrm{C}$ vacancies}

Considering that $\mathrm{ZrC}_{x}$ can be stable with a wide-range of sub-stoichiometries $(0.6<\mathrm{x}<0.98)[2]$, it is important to consider the effect of $\mathrm{C}$ vacancies $\left(\mathrm{V}_{\mathrm{C}}\right)$ on the threshold displacement energies. To explore the effect of $\mathrm{V}_{\mathrm{C}}$, initial structures were created with a $\mathrm{V}_{\mathrm{C}}$ as the nearest-neighbor of a $\mathrm{C}$ PKA along the [011] direction and as the nearest-neighbor of a Zr PKA along the [001] direction, as shown in Figs. 1 (c) and (d). The $E_{d}$ values for $\mathrm{C}$ and $\mathrm{Zr}$ along the high-symmetry directions in the presence of the $\mathrm{V}_{\mathrm{C}}$ are shown in Table 2. The largest change is increase in $E_{d}$ in $\mathrm{C}[111]$ by $4 \mathrm{eV}$. Other changes are within the error range. The biggest change is found in the case of $C[111]$ with $\mathrm{V}_{\mathrm{C}}$ because the low Ed value for this case was in part due to a bonding of the PKA C with nearest neighbors (see Sec. 3.1). The $\mathrm{V}_{\mathrm{C}}$ therefore reduces opportunities for this bonding, and increases $E_{d}$ by about $4 \mathrm{eV}$ to $11 \pm 1 \mathrm{eV}$.

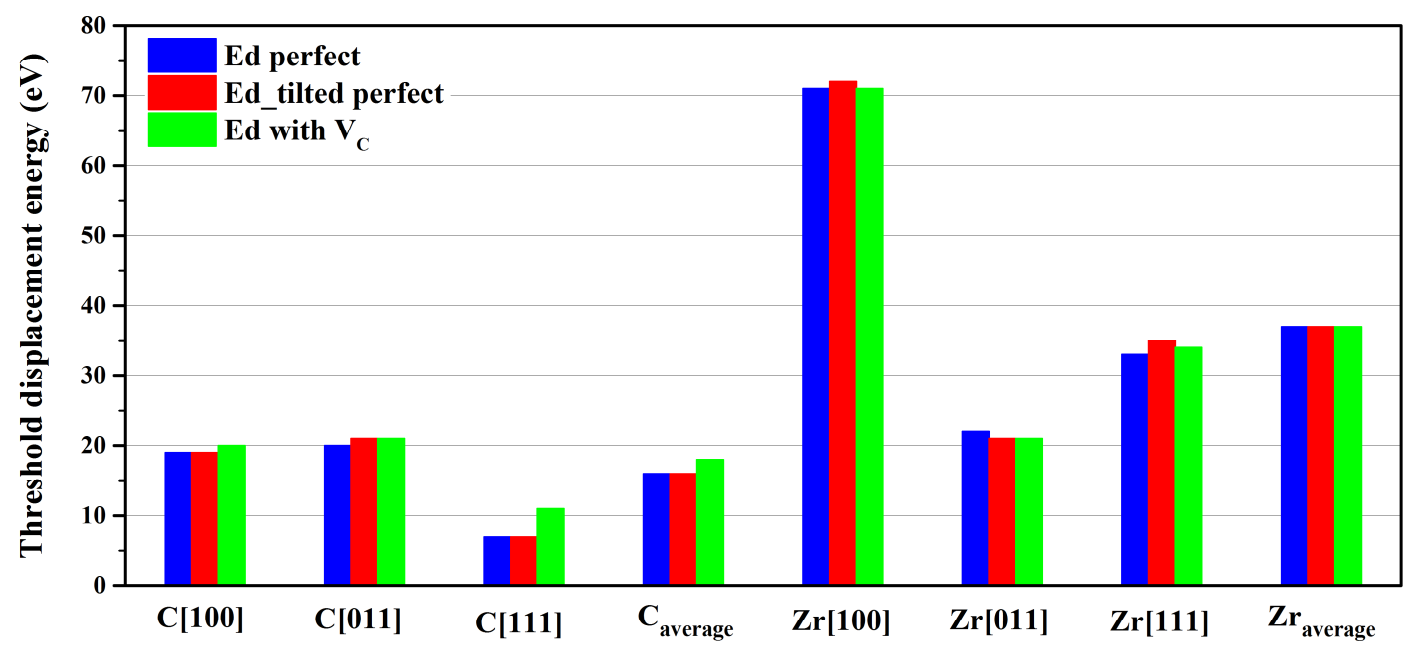

Figure 3. The $E_{d}$ values in $\mathrm{ZrC}$ for the three cases studied in this work: (i) $E_{d}$ for impulses along the high-symmetry crystallographic directions in the perfect crystals $\left(E_{d}\right.$ perfect, 
blue); (ii) $E_{d}$ for impulses tilted off the high-symmetry crystallographic directions in the perfect crystals ( $E_{d}$ _tilted perfect, red); (iii) $E_{d}$ for impulses along the high-symmetry crystallographic directions in $\mathrm{ZrC}$ in the presence of a $\mathrm{C}$ vacancy near the PKA $\left(E_{d}\right.$ with $\mathrm{V}_{\mathrm{C}}$, green).

As a summary, Fig. 3 shows the comparison of our results for the three cases studied in this work, (i) $E_{d}$ along the high-symmetry directions in the perfect crystal, (ii) $E_{d}$ along the slightly-off high-symmetry directions in the perfect crystal, and (iii) $E_{d}$ along the high-symmetry directions in the presence of $\mathrm{V}_{\mathrm{C}}$. The weighted average $E_{d}$ values along the slightly-off high-symmetry directions or in the presence of $\mathrm{V}_{\mathrm{C}}$ are equal to the corresponding value along the high-symmetry directions in the perfect crystal for $\mathrm{Zr}$, and differ slightly for $\mathrm{C}$ for in the presence of $\mathrm{V}_{\mathrm{C}}$ due to the change in $\mathrm{C}[111]$ in the presence of $\mathrm{V}_{\mathrm{C}}$.

\section{Conclusion}

We used ab initio molecular dynamics (AIMD) methods to calculate $E_{d}$ in $\mathrm{ZrC}$ along the main crystallographic directions, tilted directions, and in the presence of a $\mathrm{C}$ vacancy. The averaged threshold displacement energies along the main crystallographic directions on the $\mathrm{C}$ and $\mathrm{Zr}$ sublattices are $16 \mathrm{eV}$ and $37 \mathrm{eV}$, respectively. Our average $E_{d}$ results are smaller than the estimated average $E_{d}$ of $25 \mathrm{eV}$ for $\mathrm{C}$ and similar to the $E_{d}$ of 
$35 \mathrm{eV}$ for $\mathrm{Zr}$ reported by Gosset, et al. [5], which were estimated indirectly based on values measured in $\mathrm{TaC}$. We expect that these first principles calculations of $E_{d}$ in $\mathrm{ZrC}$ will provide a more reliable estimation than this simple extrapolation. In addition, our calculations provide detailed insight into the Frenkel pair formation mechanisms and directional dependence of $E_{d}$ in $\mathrm{ZrC}$.

The sensitivity of $E_{d}$ on the directions was investigated by tilting the directions of PKA velocities from the main crystallographic directions by a small angle. No obvious effects were found. A neighboring C vacancy can increase the values of $E_{d}$, although the effect was just $4 \mathrm{eV}$ and only along a specific direction.

Our results provide an understanding of the basic damage production processes in $\mathrm{ZrC}$. Furthermore, they provide a foundation for aiding the design and interpretation of $\mathrm{ZrC}$ irradiation experiments and support for irradiation modeling, e.g., for use in validating potentials or input to SRIM simulations.

\section{Acknowledgement}

This research is being performed using funding received from the DOE Office of Nuclear Energy's Nuclear Energy University Programs (NEUP 10-679). This work benefitted from the use of the Extreme Science and Engineering Discovery Environment (XSEDE), which is supported by National Science Foundation grant number OCI-1053575. The 
authors acknowledge Prof. Arthur Motta, Prof. Todd Allen, Mr. Christopher J. Ulmer, and Dr. Yina Huang for useful discussions.

\section{Appendix}

In the literature there are sometimes different definitions for the threshold displacement energies [12-15]. In this paper, $E_{d}$ is defined as the minimum energy required to permanently displace the same type of atom as the PKA [13-15], which we believe is the most widely used definition. Another possible definition is to define the threshold displacement energy as the minimum energy required to form a stable Frenkel pair of any type, i.e., the Frenkel pair may or may not be on the same type of lattice as the PKA [12]. We denote this alternative definition as $E_{d}$ '. For completeness Table A.1 lists $E_{d}$ ' values in $\mathrm{ZrC}$ and $\mathrm{SiC}[12]$ using this alternative definition.

\begin{tabular}{|c|c|c|c|}
\hline Direction & $\begin{array}{c}E_{d}, \text { in } \mathrm{ZrC}(\mathrm{eV}) \\
216 \text { atoms }\end{array}$ & $\begin{array}{c}E_{d}, \text { in } \mathrm{ZrC}(\mathrm{eV}) \\
64 \text { atoms }\end{array}$ & $\begin{array}{c}E_{d}, \text { in } \mathrm{SiC}(\mathrm{eV}) \\
64 \text { atoms }[12]\end{array}$ \\
\hline $\mathrm{C}[001]$ & $19 \pm 1$ & $20 \pm 1$ & $18 \pm 1$ \\
\hline $\mathrm{C}[011]$ & $20 \pm 1$ & $17 \pm 1$ & $14 \pm 1$ \\
\hline $\mathrm{C}[111]$ & $7 \pm 1$ & $39 \pm 1$ & $38 \pm 1$ \\
\hline $\mathrm{C}[\overline{1} \overline{1} \overline{1}]$ & $7 \pm 1$ & $39 \pm 1$ & $38 \pm 1$ \\
\hline $\begin{array}{l}\text { C sublattice } \\
\text { weighted average }\end{array}$ & $16 \pm 1$ & $22 \pm 1$ & $19 \pm 1$ \\
\hline
\end{tabular}




\begin{tabular}{llll}
$\operatorname{Zr}[001]$ & $18 \pm 1$ & $33 \pm 1$ & $46 \pm 1$ \\
$\operatorname{Zr}[011]$ & $22 \pm 1$ & $22 \pm 1$ & $45 \pm 1$ \\
$\operatorname{Zr}[111]$ & $27 \pm 1$ & $32 \pm 1$ & $22 \pm 1$ \\
$\operatorname{Zr}[\overline{1} \overline{1} \overline{1}]$ & $27 \pm 1$ & $32 \pm 1$ & $22 \pm 1$ \\
$\begin{array}{l}\mathrm{Zr} \text { sublattice } \\
\text { weighted average }\end{array}$ & $22 \pm 1$ & $27 \pm 1$ & $38 \pm 1$ \\
\hline \hline
\end{tabular}

\section{References}

1. L.L. Snead; Y. Katoh; S. Kondo, J. Nucl. Mater. 399 (2010) 200-207.

2. H.F. Jackson; W.E. Lee; J.M.K. Editor-in-Chief: Rudy, 2.13 - Properties and Characteristics of ZrC. In Comprehensive Nuclear Materials, Elsevier: Oxford, 2012; pp 339-372.

3. $\quad$ S. Kim; I. Szlufarska; D. Morgan, J. Appl. Phys. 107 (2010) 053521.

4. D. Gosset; M. Dolle; D. Simeone; G. Baldinozzi; L. Thome, Nucl. Instrum. Methods Phys. Res., Sect. B 266 (2008) 2801-2805.

$5 . \quad$ D. Gosset; M. Dolle; D. Simeone; G. Baldinozzi; L. Thome, J. Nucl. Mater. 373 (2008) 123-129.

6. Y. Yang; C.A. Dickerson; H. Swoboda; B. Miller; T.R. Allen, J. Nucl. Mater. 378 (2008) 341-348.

7. $\quad$ M.-J. Zheng; I. Szlufarska; D. Morgan, J. Nucl. Mater. 457 (2015) 343-351.

8. C. Jiang; M.-J. Zheng; D. Morgan; I. Szlufarska, Phys. Rev. Lett. 111 (2013) 155501.

9. J. Gan; M.K. Meyer; R.C. Birtcher; T.R. Allen, ASTM Spec. Tech. Publ. (2005).

10. L. Van Brutzel; J.P. Crocombette, Nucl. Instrum. Methods Phys. Res., Sect. B 255 (2007) 141-145.

11. J.F. Ziegler; M.D. Ziegler; J.P. Biersack, Nucl. Instrum. Methods Phys. Res., Sect. B 268 (2010) 1818-1823.

12. G. Lucas; L. Pizzagalli, Phys. Rev. B 72 (2005) R161202.

13. F. Gao; H. Xiao; X. Zu; M. Posselt; W.J. Weber, Phys. Rev. Lett. 103 (2009) 027405.

14. F. Gao; H.Y. Xiao; W.J. Weber, Nucl. Instrum. Methods Phys. Res., Sect. B 269 (2011) 1693-1697. 
15. M. Robinson; N.A. Marks; K.R. Whittle; G.R. Lumpkin, Phys. Rev. B 85 (2012) 104105.

16. G.S. Was, Fundamentals of Radiation Materials Science: Metals and Alloys. Springer: Berlin, 2007.

17. K. Trachenko, Journal of Physics-Condensed Matter 16 (2004) R1491-R1515.

18. J. Nord; K. Nordlund; J. Keinonen, Phys. Rev. B 68 (2003) 184104.

19. B. Park; W.J. Weber; L.R. Corrales, Nucl. Instrum. Methods Phys. Res., Sect. B 166 (2000) 357-363.

20. H.Y. Xiao; F. Gao; X.T. Zu; W.J. Weber, J. Appl. Phys. 105 (2009) 123527.

21. G. Lucas; L. Pizzagalli, Nucl. Instrum. Methods Phys. Res., Sect. B 229 (2005) 359-366.

22. H.Y. Xiao; F. Gao; W.J. Weber, J. Phys.: Condens. Matter 22 (2010) 415801.

23. G. Kresse; J. Furthmuller, Computational Materials Science 6 (1996) 15-50.

24. G. Kresse; J. Furthmuller, Phys. Rev. B 54 (1996) 11169-11186.

25. G. Kresse; J. Hafner, Phys. Rev. $B 47$ (1993) 558-561.

26. G. Kresse; J. Hafner, Phys. Rev. B 49 (1994) 14251-14269.

27. J.P. Perdew; K. Burke; M. Ernzerhof, Phys. Rev. Lett. 77 (1996) 3865-3868.

28. L.R. Corrales; A. Chartier; R. Devanathan, Nucl. Instrum. Methods Phys. Res., Sect. B 228 (2005) 274-281.

29. H. Inui; H. Mori; A. Suzuki; H. Fujita, Phil. Mag. B 65 (1992) 1-14.

30. M. Ishimaru; I.-T. Bae; Y. Hirotsu, Phys. Rev. B 68 (2003) 144102.

31. L.L. Snead; J.C. Hay, J. Nucl. Mater. 273 (1999) 213-220.

32. W.J. Weber; L.M. Wang, Nucl. Instrum. Methods Phys. Res., Sect. B 106 (1995) 298-302. 